\title{
Os índices de trincas e fraturas em laminados cerâmicos são por razões oclusais?
}

\section{Uma revisão sistemática}

\author{
Are the rates of cracks and fractures in ceramic veneers for occlusal reasons? A systematic review \\ ¿Son las tasas de grietas y fracturas en los carillas cerámicas por razones oclusales? Una revisión \\ sistemática
}

Recebido: 03/04/2021 | Revisado: 12/04/2021 | Aceito: 14/04/2021 | Publicado: 28/04/2021

Jéssica Monique Lopes Moreno

ORCID: https://orcid.org/0000-0002-8808-4872 Universidade Estadual "Júlio de Mesquita Filho", Brasil

E-mail: jessica_morenoo@hotmail.com

Danila de Oliveira

ORCID: https://orcid.org/0000-0001-7788-0243

Universidade Estadual “Júlio de Mesquita Filho", Brasil

E-mail: danila.oliveira@ unesp.br

Paulo Sergio Morais Sales

ORCID: https://orcid.org/0000-0003-1017-8665 Universidade Brasil, Brasil

E-mail: paulomsales@hotmail.com

Caroline Liberato Marchiolli

ORCID: https://orcid.org/0000-0001-8881-4882 Universidade Brasil, Brasil

E-mail: caaliberato@gmail.com

Bruno Mendes Tenório

ORCID: https://orcid.org/0000-0002-8959-695X Universidade Estadual “Júlio de Mesquita Filho", Brasil

E-mail: brunocoelho.mnds@gmail.com

Ariane Rodrigues Barion

ORCID: https://orcid.org/0000-0002-4181-8947 Universidade Estadual “Júlio de Mesquita Filho", Brasil

E-mail: barionariane@gmail.com

Ricardo Alves Toscano

ORCID: https://orcid.org/0000-0002-6381-463X Universidade Estadual “Júlio de Mesquita Filho", Brasil E-mail: odontosc.joi@terra.com.br

Eduardo Passos Rocha

ORCID: https://orcid.org/0000-0002-0157-3690 Universidade Estadual “Júlio de Mesquita Filho", Brasil E-mail: 1rocha.eduardo@gmail.com

Wirley Gonçalves Assunção

ORCID: https://orcid.org/0000-0002-8903-0737 Universidade Estadual “Júlio de Mesquita Filho", Brasil E-mail: wirley.assuncao@unesp.br

\begin{abstract}
Resumo
Objetivo: avaliar, a partir de uma revisão sistemática, qual o índice de trincas e fraturas em laminados cerâmicos, a fim de estabelecer as principais causas para essas falhas. Metodologia: Foi realizada uma revisão sistemática de estudos clínicos prospectivos e retrospectivos que analisaram o índice de falhas por trincas ou fratura da restauração de dentes submetidos ao tratamento com laminados cerâmicos. Utilizou-se os descritores "ceramic" e "veneer" adaptados de acordo com as bases de dados consultadas: Science Direct, LILACS, MEDLINE (PubMed) e Google Scholar. Inicialmente foram encontrados 755 artigos, após a leitura e aplicação dos critérios de elegibilidade e exclusão, 4 artigos foram selecionados para a análise quantitativa e 5 para a análise qualitativa. Esta revisão seguiu as recomendações do Preferred Reporting Items for Systematic Reviews and Meta-analyzes (PRISMA). Resultados: A descoloração marginal foi a falha mais comumente encontrada $(17,2 \%)$, seguida por lesão periodontal ou gengival $(12,8 \%)$, soltura da peça $(4,4 \%)$, fratura da cerâmica $(2,7 \%)$ e trincas da cerâmica $(1,7 \%)$. As falhas por lesão endodôntica, cárie secundária, fratura do dente e alteração de cor da cerâmica foram menores que $1 \%$. Conclusão: A atenção em relação a oclusão, seus estudos e conceitos, devem ser levados em consideração em todo o processo do tratamento com laminados cerâmicos para garantir a longevidade e sucesso clínico.
\end{abstract}

Palavras-chave: Cerâmica; Oclusão dentária; Falha de restauração dentária. 


\begin{abstract}
Objective: to assess, based on a systematic review, what the crack and fracture rate in ceramic veneer is, in order to establish the main causes for these failures. Methodology: A systematic review of prospective and retrospective clinical studies that analyzed the failure rate due to cracks or fractures in the restoration of teeth restored with ceramic veneers was carried out. The descriptors "ceramic" and "veneer" were used, adapted according to the databases consulted: Science Direct, LILACS, MEDLINE (PubMed) and Google Scholar. Initially, 755 articles were found, after reading and applying the eligibility and exclusion criteria, 4 articles were selected for the quantitative and 5 for the qualitative analyses. This review followed the recommendations of the Preferred Reporting Items for Systematic Reviews and Meta-analyzes (PRISMA). Results: Marginal discoloration was the most commonly found failure $(17.2 \%)$, followed by periodontal or gingival inflammation $(12.8 \%)$, debonding $(4.4 \%)$, crack of ceramic $(2.7 \%)$ and fracture of ceramic $(1.7 \%)$. Failures due to endodontic injury, secondary caries, tooth fracture and color change of the ceramic were less than 1\%. Conclusion: Attention to occlusion, its studies and concepts, must be taken into account in the entire process of treatment with ceramic veneers to ensure longevity and clinical success.
\end{abstract}

Keywords: Ceramics; Dental occlusion; Dental restoration failure.

\title{
Resumen
}

Objetivo: evaluar, a partir de una revisión sistemática, cuál es la tasa de grieta y fractura en las carillas cerámicas, con el fin de establecer las principales causas de estos fallos. Metodología: Se realizó una revisión sistemática de estudios clínicos prospectivos y retrospectivos que analizaron la tasa de falla por grieta o fracturas en la restauración de dientes restaurados con carillas cerámicas. Se utilizaron los descriptores “cerámica” y "carilla”, adaptados según las bases de datos consultadas: Science Direct, LILACS, MEDLINE (PubMed) y Google Scholar. Inicialmente se encontraron 755 artículos, luego de leer y aplicar los criterios de elegibilidad y exclusión, se seleccionaron 4 artículos para los análisis cuantitativos y 5 para los cualitativos. Esta revisión siguió las recomendaciones de los elementos de informe preferidos para revisiones sistemáticas y metanálisis (PRISMA). Resultados: la decoloración marginal fue la falla más comúnmente encontrada (17.2\%), seguida de la inflamación periodontal o gingival (12.8\%), el desprendimiento (4.4\%), el agrietamiento de la cerámica $(2.7 \%)$ y la fractura de la cerámica (1.7\%). Las fallas por lesión endodóntica, caries secundaria, fractura dentaria y cambio de color de la cerámica fueron menores al $1 \%$. Conclusión: La atención a la oclusión, sus estudios y conceptos, debe tenerse en cuenta en todo el proceso de tratamiento con carillas cerámicas para asegurar la longevidad y el éxito clínico.

Palabras clave: Cerámica; Oclusión dental; Fracaso de la restauración dental.

\section{Introdução}

Os laminados cerâmicos vêm sendo rotineiramente indicados para o fechamento de diastemas, correções de pequenos maus posicionamentos, desgastes dentários e para alterações de formato e cor dos dentes (Alothman \& Bamasoud, 2018; Layton \& Clarke, 2013). O tratamento é considerado minimamente invasivo, uma vez que pouco ou nenhum desgaste da estrutura dentária é necessário para permitir o correto assentamento da peça sobre o dente. Além disso, com o avanço das técnicas adesivas de cimentação e a melhora nas características micro-estruturais das cerâmicas utilizadas para fabricação da restauração, é possível estabelecer uma força de adesão muito confiável entre o laminado cerâmico e o esmalte dentário (Granell-Ruiz et al., 2010; Gresnigt et al., 2019; Peumans, Van Meerbeek, Lambrechts, \& Vanherle, 2000). Como resultado, estudos apontam taxas de sobrevida deste tipo de tratamento variando entre 80 a $96 \%$ após 10 a 21 anos de acompanhamento (Beier, Kapferer, \& Dumfahrt, 2012; Layton \& Walton, 2012; Rinke, Lange, \& Ziebolz, 2013; Smales \& Etemadi, 2004). Para alguns autores, pacientes com hábitos parafuncionais como o bruxismo, oclusão do tipo "topo a topo", higiene oral insatisfatória e esmalte dentário insuficiente podem ser considerados como fatores desfavoráveis para indicação deste tratamento (Hui, Williams, Davis, \& Holt, 1991; Sheets \& Taniguchi, 1990).

Em uma revisão de literatura realizada por Peumans et al (2000) incluindo 13 ensaios clínicos, foi relatada uma taxa de falha devido a soltura ou fratura considerada baixa na maioria dos estudos analisados, isto é, entre 0 e $5 \%$ em um período de até 10 anos de acompanhamento. Em 3 dos estudos incluídos na revisão foram encontradas taxas de falha maiores (7, 13 e 14\%). Os autores relacionaram esse alto índice de falhas desses 3 estudos a pacientes com oclusão desfavorável, pois as restaurações foram indicadas para restabelecer a função e estética de dentes fraturados e desgastados devido ao bruxismo. Laminados cerâmicos cimentados sobre dentes com grandes superfícies de dentina exposta ou dentes com restaurações de 
resina composta preexistentes, além da experiência clinica do profissional também foram fatores associados às falhas encontradas (Peumans et al., 2000).

Na revisão realizada por Sadowsky (2006), a incidência de falha irreparável foi de até 7\% em um período de mais de 9 anos de acompanhamento, sendo que as principais falhas foram: fratura do laminado, micro-infiltração e soltura da peça. A necessidade de intervenção sem substituição da peça foi relatada como sendo de até $36 \%$ em até 10 anos de acompanhamento. Os autores também relacionaram como fatores predisponentes para a ocorrência de fraturas a adesão predominantemente em dentina, presença de restaurações em resina composta, dentes tratados endodonticamente e carga funcional ou parafuncional excessiva (Sadowsky, 2006).

Já para Calamia et al (2007), o problema mais comum em laminados cerâmicos é a falha coesiva da própria cerâmica que pode ocorrer devido a uma restauração mal ajustada ou pelo uso de um cimento resinoso muito viscoso na etapa de cimentação ou, ainda, como resultado de uma oclusão traumática ou patológica (Calamia \& Calamia, 2007). Apesar da diferença dos períodos de acompanhamento e forma de análise dos estudos analisados, todos relacionaram a oclusão mais próxima do ideal possível e o manejo de hábitos parafuncionais, como o bruxismo, como fator crucial para o sucesso e longevidade do tratamento com laminados cerâmicos. Sendo assim, o objetivo do presente estudo foi avaliar, a partir de uma revisão sistemática, qual o índice de trincas e fraturas em laminados cerâmicos, a fim de estabelecer as principais causas para essas falhas.

\section{Metodologia}

Os critérios para a construção desta revisão sistemática foram definidos com base no Preferred Reporting Items for Systematic Reviews and Meta-Analyses (Moher, Liberati, Tetzlaff, \& Altman, 2009), além da metodologia realizada por Layton, 2013 (Layton \& Clarke, 2013) em sua revisão sistemática e meta-análise. Foi norteador para essa pesquisa o desejo de responder e relacionar numa dinâmica efeito-causa a questão-alvo seguinte: "Qual o índice de fraturas e trincas em laminados cerâmicos?"

\subsection{Critérios de Elegibilidade e estratégias de busca}

Foi realizada uma série de pesquisas sistemáticas no banco de dados de quatro indexadores de conteúdo acadêmico: Science Direct, LILACS, MEDLINE (PubMed) e Google Scholar. As buscas foram realizadas por dois pesquisadores independentes nas bases de dados selecionadas até o dia 7 de julho de 2020. Primeiramente, foi feita a busca com os termos "ceramic" e "veneers". A estratégia de utilização dos descritores foi readequada para cada uma das bases de dados pesquisadas. Foi realizada, ainda, uma busca manual nas referências dos artigos selecionados. Foram incluídos estudos sem restrição de idioma, período ou localização.

Para a análise da questão-alvo, a pesquisa foi estruturada na forma PICOS, ou seja, População, Intervenção, Comparação, Outcomes (desfecho) e Study design. A população de interesse foi considerada como pacientes adultos com ou sem comorbidades; intervenção: dentes submetidos a tratamento com laminados cerâmicos; comparação: inexistente para este estudo; outcomes: índice de falhas por trincas ou fratura da restauração; study design: estudos clínicos prospectivos e longitudinais e estudos de retrospectiva clínica.

\subsection{Critérios de exclusão}

Foram excluídos da revisão os seguintes estudos:

1. Não se enquadrava como prospectivo ou retrospectiva clínica em laminados cerâmicos;

2. Não foi especificado o(s) tipo(s) de cerâmica(s) utilizada(s); 
3. Foram realizados para comparar duas e/ou marcas e materiais;

4. Inferiores a três anos da amostragem inicial;

5. Restaurações realizadas exclusivamente em dentes não vitais;

6. Estudos com conflitos de interesses;

\subsection{Seleção dos estudos}

A partir da busca nas bases de dados foram encontrados um total de 755 artigos, dos quais 723 foram excluídos através de seus títulos e resumos, restando 32 artigos relacionados ao objetivo desse estudo (Figura 1). Após a leitura completa, excluiu-se 28 estudos. Como resultado final foram eleitos 4 estudos destinados à análise quantitativa (Tabela 1). Dentre os artigos excluídos da análise quantitativa, foi possível aplicar 4 dos 6 critérios de exclusão definidos (em ordem decrescente de ocorrência dos critérios: 1 - 19 artigos; 5 - 6 artigos; 3 - 2 artigos; 2 - 1 artigo). Quando um estudo excluído era considerado de relevância para a discussão do assunto, este era incluído para análise qualitativa (Tabela 2).

Figura 1. Fluxograma de busca e análise dos artigos.

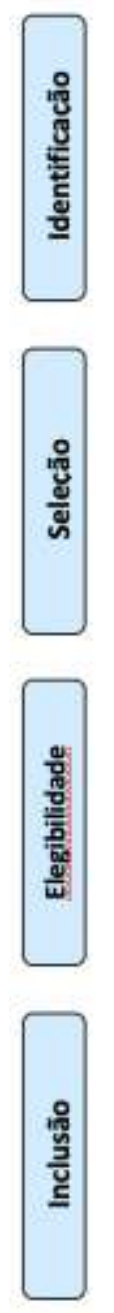

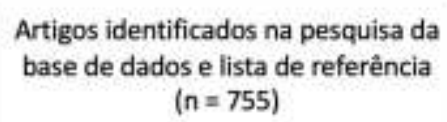

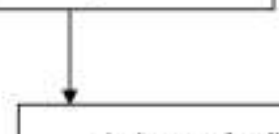

Artigos após eliminar os duplicados ( $n=755$ )
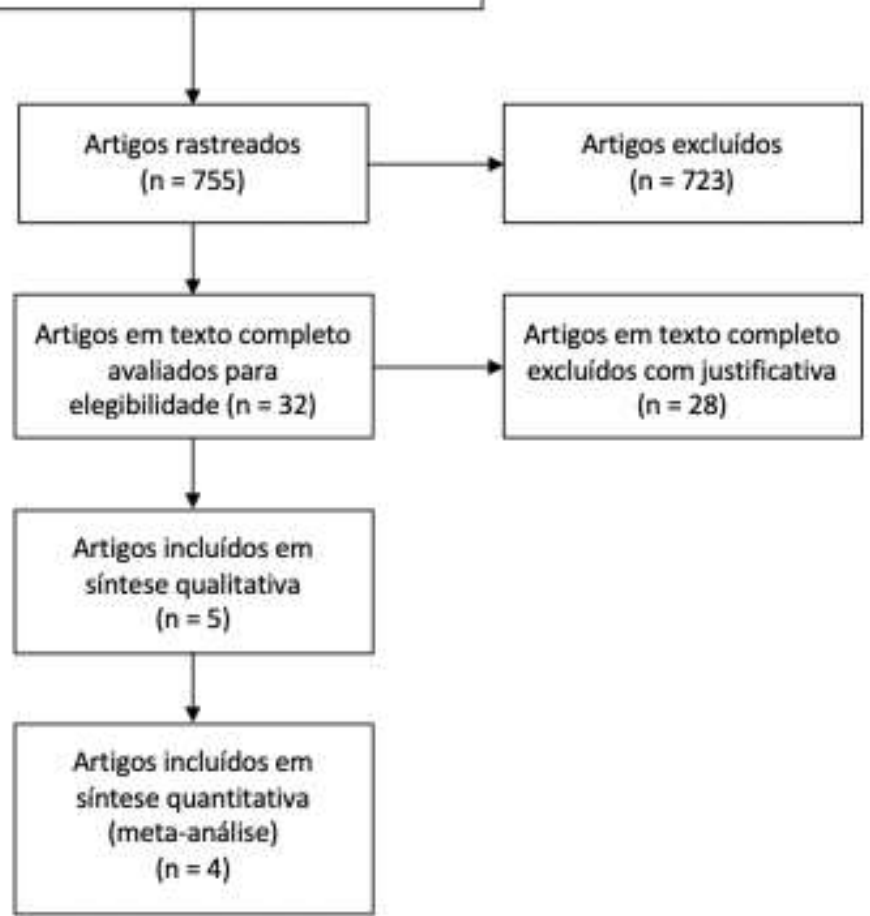

Fonte: Autores (2021). 
Tabela 1. Estudos incluídos para análise quantitativa.

\begin{tabular}{|c|c|}
\hline Autor/ano & Descrição do estudo \\
\hline Beier et al 2012 & $\begin{array}{l}\text { Retrospectiva clínica; } \\
\text { Tempo: } 20 \text { anos; } \\
\text { Período de inclusão: } 1987-2009 \text {; } \\
\text { Amostra: } 318 \text { laminados cerâmicos em } 84 \text { pacientes. ( } 38 \text { homens, } 46 \text { mulheres) } \\
\mu \cong 3,8 \text { laminados cerâmicos/paciente* }\end{array}$ \\
\hline Granell-Ruiz et al 2010 & $\begin{array}{l}\text { Estudo clínico longitudinal; } \\
\text { Tempo: } 3-11 \text { anos; } \\
\text { Período de inclusão: } 1995-2003 \text {; } \\
\text { Amostra: } 323 \text { laminados cerâmicos em } 70 \text { pacientes. ( } 17 \text { homens, } 53 \text { mulheres) Notamos que a } \\
\text { maioria dos pacientes havia entre } 31 \text { e } 40 \text { anos. } \\
\mu \cong 4,6 \text { laminados cerâmicos/paciente* }\end{array}$ \\
\hline Gresnigt et al 2019 & $\begin{array}{l}\text { Estudo clínico prospectivo; } \\
\text { Tempo: } 11 \text { anos; } \\
\text { Período de inclusão: } 2007-2018 \text {; } \\
\text { Amostra: } 444 \text { laminados cerâmicos em } 118 \text { pacientes. ( } 38 \text { homens, } 80 \text { mulheres) Média de idade: } \\
42,1 \text { anos. } \\
\mu \cong 3,8 \text { laminados cerâmicos/paciente* }\end{array}$ \\
\hline Rinke et al 2012 & $\begin{array}{l}\text { Retrospectiva clínica; } \\
\text { Tempo: } 3 \text { anos; } \\
\text { Período de inclusão: } 2002-2008 \text {; } \\
\text { Amostra: } 130 \text { laminados cerâmicos em } 37 \text { pacientes. ( } 16 \text { homens, } 21 \text { mulheres) Média de idade: } \\
46.1 \text { a } 12.7 \text { sendo a mais nova com } 23 \text { anos e o mais velho com } 70 \text { anos de idade. } \\
\mu \cong 3,5 \text { laminados cerâmicos/paciente* }\end{array}$ \\
\hline
\end{tabular}

*: Dados não fornecidos pelo estudo original.

Fonte: Autores (2021).

Tabela 2. Estudos excluídos, porém elegíveis para análise qualitativa.

\begin{tabular}{ll}
\hline Autor & Critério(s) de exclusão \\
\hline Albanesi et al 2016 & 1 \\
\hline Alhekeir et al 2014 & 1 \\
\hline Layton et al 2013 & 1 \\
\hline Nejatidanesh et al 2018 & 3 \\
\hline Schmidt et al 2011 & 1 \\
\hline
\end{tabular}

Fonte: Autores.

\section{Resultados}

Nesse estudo foram incluídos artigos desenvolvidos em três períodos diferentes de tempo de avaliação clínica. O primeiro sendo após três anos de instalação dos laminados cerâmicos, o segundo onze anos seguidos do procedimento, e o terceiro vinte anos passados da confecção das restaurações. As falhas com suas respectivas incidências podem ser observadas na Tabela 3 . 
Tabela 3. Falhas mais comuns nos laminados cerâmicos.

\begin{tabular}{|c|c|c|c|c|c|}
\hline Tipo de falha & $\begin{array}{l}\text { Beier et al } \\
2012 *\end{array}$ & $\begin{array}{l}\text { Granell-Ruiz et } \\
\text { al } 2010\end{array}$ & $\begin{array}{l}\text { Grasnigt et al } \\
2019\end{array}$ & Rinke et al 2012 & $\begin{array}{l}\text { Total } \\
n=1.195\end{array}$ \\
\hline $\begin{array}{l}\text { Descoloração } \\
\text { marginal }\end{array}$ & 0 & $127(39,3 \%)$ & $71(16,0 \%)$ & $8(6,1 \%)$ & $206(17,2 \%)$ \\
\hline $\begin{array}{l}\text { Lesão gengival ou } \\
\text { periodontal }\end{array}$ & $82(25,8 \%)$ & $70(21,7 \%)$ & 0 & $1(<1 \%)$ & $153(12,8 \%)$ \\
\hline Soltura & $2(<1 \%)$ & $29(9,0 \%)$ & $19(4,2 \%)$ & $3(2,3 \%)$ & $53(4,4 \%)$ \\
\hline $\begin{array}{l}\text { Fraturas da } \\
\text { cerâmica }\end{array}$ & $13(4,3 \%)$ & $13(4,0 \%)$ & $3(<1 \%)$ & $4(3,0 \%)$ & $33(2,7 \%)$ \\
\hline Trincas da cerâmica & $6(2,0 \%)$ & 0 & $14(3,1 \%)$ & $1(<1 \%)$ & $21(1,7 \%)$ \\
\hline Lesão endodôntica & 0 & $9(2,8 \%)$ & $1(<1 \%)$ & $2(1,5 \%)$ & $12(1,0 \%)$ \\
\hline Cáries secundárias & 0 & $10(3,1 \%)$ & $1(<1 \%)$ & 0 & $11(1,0 \%)$ \\
\hline Fraturas do dente & $2(<1 \%)$ & 0 & $3(<1 \%)$ & 0 & $5(<1 \%)$ \\
\hline $\begin{array}{l}\text { Alteração de cor da } \\
\text { cerâmica }\end{array}$ & 0 & $2(<1 \%)$ & $1(<1 \%)$ & 0 & $3(<1 \%)$ \\
\hline
\end{tabular}

* Foram consideradas 298 restaurações, devido a 20 falhas ocorridas antes do período de avaliação.

Fonte: Autores (2021).

A falha mais comum encontrada nos estudos foi a descoloração marginal, representando 17,2\% da amostra total $(n=1.195)$. Alguns estudos ainda classificavam essa falha em níveis de urgência para correção, como o demonstrado por Gresnigt et al (2019) através do protocolo do United States Public Health Service (USPHS). Apesar de ser a causa mais comum dos estudos comparados, ela não foi relatada por Beier et al (2012).

A presença de lesão periodontal/gengival nos pacientes incluídos foi a segunda falha mais frequente, sendo o estudo de Beier et al (2012) o de maior prevalência. Apesar de Rinke et al (2012) ter relatado apenas um caso dessa falha, foi também a única que acarretou a perda do elemento dentário por lesão periodontal dentre todos os pacientes do estudo ( $\mathrm{n}=1.195$ ), o que vai contra o fato de também representar o período de menor retrospectiva clínica. Granell-Ruiz et al (2010) foi o único estudo que relacionou o sangramento gengival observado com a recessão gengival em escalas de 0 a 3 .

A soltura da peça constou em terceiro lugar na lista de falhas observadas. O estudo de Granell-Ruiz et al (2010), em que esta foi mais prevalente, levou em consideração a opinião da literatura, que mostra ser mais comum a soltura em dentes com pouco remanescente de esmalte ou restaurados com resina composta. Gresnigt et al afirma que os achados em soltura no seu estudo vão de encontro com o que é constatado em outras pesquisas em que, de acordo com este, seria uma falha comum nos primeiros quatro anos de retrospectiva clínica, também sendo observado por Rinke et al (2012). Os dois estudos restantes não apresentaram outros dados relevantes sobre a ocorrência da soltura em seus achados.

Em todos os estudos analisados, a oclusão foi considerada um fator que pode acarretar o aumento ou redução de falhas dos laminados cerâmicos. O quarto e quinto itens de maior falha nas restaurações foram, respectivamente, fraturas e trincas da cerâmica. Dos estudos aqui relacionados, Beier et al (2012) e Gresnigt et al (2019) foram os que obtiveram maior prevalência dessas falhas. Na sua leitura, percebe-se que ambos relacionaram a maior incidência de fraturas com a presença de pacientes portadores de bruxismo dentre a amostra em seus estudos, assim como foi o caso de Granell-Ruiz et al (2010). Isso poderia ser verificável quando em comparação a pesquisa de Rinke et al (2012), que excluiu totalmente pacientes com bruxismo da sua avaliação, reduzindo altamente o número de fraturas e rachaduras reportadas, mesmo em pouco período de tempo clínico, haja vista que isso não seria de tanta relevância, já que Beier et al (2012) possui casos com tempos de preservação parecidos em seu estudo. 


\section{Discussão}

Como a maioria dos laminados cerâmicos instalados se encontram nos dentes anteriores da maxila e na superfície vestibular, é comum se pensar que a oclusão não é um fator importante para o sucesso das restaurações. Porém, na era da prática baseada em evidências, a literatura diz o contrário (Calamia \& Calamia, 2007; Carlsson, 2010). Um correto restabelecimento da dimensão vertical, além do guia canino e incisivo, pode ajudar na preservação do trabalho realizado e da saúde bucal como um todo. A diferença na rigidez do material empregado, e em constante contato com a superfície dos dentes anteriores mandibulares hígidos pode trazer consequências periodontais, como sinais de inflamação e até mesmo recessão gengival não esperada, principalmente em regiões de caninos e pré-molares que foram inadvertidamente submetidos a um aumento do estresse oclusal após a instalação das restaurações (Calamia \& Calamia, 2007).

É importante ressaltar, ainda, os altos índices de recessão gengival causados por má oclusão em dentes anteriores inferiores (Kundapur, Bhat, \& Bhat, 2009). Essa ocorrência, que sozinha já configura um quadro de piora no diagnóstico, pode ser agravada dependendo do tipo de cerâmica utilizada no arco antagonista, haja vista que o contato de texturas tão diferentes diretamente com o esmalte pode causar ou agravar danos irreversíveis (Calamia \& Calamia, 2007).Essa teoria vai de encontro como as altas taxas de sangramento gengival encontradas nos estudos de Granell-Ruiz et al (2010), principalmente nas áreas onde foi relatado recessão gengival. Na discussão, foram utilizados dados de outros estudos para justificar essa ocorrência, que estaria associada ao envelhecimento fisiológico. Entretanto, uma análise oclusal mais profunda, associada com a revisão de higiene do paciente, considerando o índice de sangramentos ativos, poderia ter sido considerada, incluindo-se nessa indicação os estudos de Beier et al (2012) e Rinke et al (2012). Não somente no pré-operatório, como foi realizado em ambos. Em procedimentos reabilitadores como os laminados cerâmicos, deve existir um planejamento criterioso, almejando características como a reconstrução de guia anterior para os movimentos excêntricos da mandíbula (Badel, Marotti, Pavicin, \& Basić-Kes, 2012). A elaboração de esquemas de desoclusão tem mostrado que os casos mais comuns envolvem lateralidade guiada por caninos, ou caninos e pré-molares. Isso também deixa esses grupos mais expostos ao desgaste fisiológico que acompanha o envelhecimento (Abduo, Tennant, \& McGeachie, 2013).

Já a soltura, terceira maior causa de falhas, foi mais comum nos estudos de Granell-Ruiz et al (2010) e Gresnigt et al (2019), sendo menos frequente no segundo. Nos dois estudos, essa falha foi atribuída à grande parte das restaurações terem sido cimentadas sobre dentina e/ou material restaurador, e não em esmalte. Porém, ainda que não seja possível evitar essa falha quando a restauração se encontra em área com mais de 50\% de dentina, estudos mostraram que o selamento imediato da dentina faz com que esse substrato seja mais aceitável para cimentação (Gresnigt et al., 2019). A literatura concorda quanto ao fato de que restaurações com adesão em esmalte, garantem uma menor chance de soltura (Layton \& Clarke, 2013). Porém, mesmo sendo o cenário ideal, acaba sendo também, raro por conta da realidade clínica que se encontram os pacientes que necessitam de reabilitação (Alhekeir, Al-Sarhan, \& Al Mashaan, 2014; Schmidt, Chiayabutr, Phillips, \& Kois, 2011). Neste sentido, em testes realizados com cerâmicas confeccionadas por sistema CAD/CAM, esse resultado não foi diferente. Apesar da menor distorção devido a precisão do processo, os resultados referentes a cimentação no período avaliado foram semelhantes (Gresnigt et al., 2019; Gurel, Sesma, Calamita, Coachman, \& Morimoto, 2013). Restaurações aplicadas em dentes que sofreram desgaste por atrição, abrasão ou erosão apresentam porções de esmalte mais finas, dificultando a adesividade (Nejatidanesh, Savabi, Amjadi, Abbasi, \& Savabi, 2018).

A quarta e quinta falhas mais comuns encontradas em laminados cerâmicos foram, respectivamente, fratura e trinca da cerâmica, com maior prevalência nos estudos de Beier et al (2012) e Granell-Ruiz et al (2010), respectivamente. A literatura afirma que, inevitavelmente ao menos $50 \%$ das restaurações indiretas envolvendo cerâmica irão falhar em algum momento da proservação clínica (Denissen, Wijnhoff, Veldhuis, \& Kalk, 1993). Fraturas e trincas ocorrem pelo acúmulo de estresse sofrido pelo material através das forças mastigatórias, o que torna essencial um correto ajuste oclusal, associado ao formato do 
preparo, que garantirá uma melhor ou pior distribuição das forças (Denissen et al., 1993; Schmidt et al., 2011). Isso pode ser explicado, ainda, quando restaurações são posicionadas majoritariamente sobre dentina, pela diferença no módulo de elasticidade desta em relação ao esmalte, sendo o da primeira mais baixo, aumentando riscos de fraturas ou trincas (Nejatidanesh et al., 2018). Rinke et al (2012), apesar da quantidade de falhas por fratura no curto período de tempo de proservação, se enquadrou dentro dos padrões encontrados na literatura, para o tipo de cerâmica utilizada em sua reabilitação. O estudo também relatou que o formato dos seus preparos foi confeccionado de certa forma, que não houvesse contato oclusal acima das bordas da restauração, o que em sua concepção aumentaria a longevidade e reduziria o risco de trincas e fraturas.

A hipótese encontrada em outros dois artigos incluídos na meta-análise foi a influência dos formatos de preparo realizados, haja vista o alto período de avaliação clínica dos 3 estudos que mais se encontraram fraturas e rachaduras. Beier et al (2012), por exemplo, em seus pacientes incluídos, realizou preparos em períodos anteriores a cinco, dez, quinze e vinte anos antes do seu estudo. Isso faz com que, invariavelmente ocorra alteração de técnicas e entendimento para cada caso atendido. Já Granell-Ruiz et al atribuiu o índice de fraturas ao formato em chanfrado palatino.

Em um estudo in vitro, conduzido por Schmidt et al (2011), foram coletados dados quanto ao suporte de forças em restaurações com dois diferentes designs de preparo, aplicados em dentes com desgaste e sem desgaste, o que seria semelhante ao processo de atrição, tanto fisiológico quanto patológico, sofrido pelos pacientes. Os achados mostraram que designs com chanfrado palatino reduziram expressivamente a suscetibilidade à fraturas da cerâmica, criando um efeito de envelope entre a restauração e o dente proporcionado pela projeção de cerâmica na face palatina, melhorando a distribuição de forças, sendo esse resultado corroborado por outros estudos (Alothman \& Bamasoud, 2018).

Comparando os resultados do estudo in vitro com um estudo clínico, percebe-se algumas diferenças consideráveis. Nos achados clínicos relatados na revisão sistemática e meta-análise de Albanesi et al (2016), os dados se mostraram inconclusivos. Enquanto de um lado corrobora-se os fatos expressos no parágrafo anterior, por outro se considera o chanfrado palatino desnecessário e mais suscetível a formação de rachaduras nessa região, com consequente fratura sob força mastigatória (Gurel et al., 2013). O único consenso encontrado na literatura sobre assunto, se trata da necessidade de avaliação oclusal minuciosa para determinação do design a ser confeccionado, tentando compatibilizar com a estética pretendida (Albanesi, Pigozzo, Sesma, Laganá, \& Morimoto, 2016; Alothman \& Bamasoud, 2018; Schmidt et al., 2011).

A existência de avaliação oclusal satisfatória, que preencha todos os requisitos listados acima, tem relação diretamente proporcional com o conhecimento dos profissionais, tanto clínicos gerais quanto especialistas, sobre a biomecânica da oclusão e sua interação com a articulação temporomandibular. Nenhum profissional ou estudante conseguirá realizar as etapas de diagnóstico das desordens temporomandibulares sem o correto embasamento teórico e treinamento clínico (O'Carroll, Leung, Fine, Boniface, \& Louca, 2019; Racich, 2018). Nos resultados obtidos, foi observado que em todos os trabalhos que envolviam pacientes bruxistas, houve maior índice de fraturas e trincas, de frequência variável para cada tipo de cerâmica escolhida na restauração, e também associadas ao uso, ou não, de dispositivos para proteção das restaurações (Beier, Kapferer, Burtscher, \& Dumfahrt, 2012; Granell-Ruiz et al., 2010; Gresnigt et al., 2019).

\section{Considerações Finais}

Existem muitas variáveis observadas nos estudos utilizados como referência, sendo a heterogeneidade no planejamento dos laminados cerâmicos a maior delas, dificultando uma comparação adequada com o disposto na literatura, o que acaba limitando as conclusões sobre o real índice de fraturas e trincas de maneira geral nesses procedimentos. No entanto, a atenção em relação a oclusão, seus estudos e conceitos, devem ser levados em consideração em todo o processo do tratamento para garantir a longevidade e sucesso clínico. 
Com um maior número de pesquisas clínicas com acompanhamento longitudinal sobre laminados cerâmicos será possível realizar futuras revisões sistemáticas com amostras maiores e maior tempo de acompanhamento que permitam não somente a quantificação dos dados coletados por análises estatísticas, mas também comparações mais detalhadas com os resultados já dispostos na literatura.

\section{Referências}

Abduo, J., Tennant, M., \& McGeachie, J. (2013). Lateral occlusion schemes in natural and minimally restored permanent dentition: a systematic review. $J$ Oral Rehabil, 40(10), 788-802. doi:10.1111/joor.12095

Albanesi, R. B., Pigozzo, M. N., Sesma, N., Laganá, D. C., \& Morimoto, S. (2016). Incisal coverage or not in ceramic laminate veneers: A systematic review and meta-analysis. J Dent, 52, 1-7. doi:10.1016/j.jdent.2016.06.004

Alhekeir, D. F., Al-Sarhan, R. A., \& Al Mashaan, A. F. (2014). Porcelain laminate veneers: Clinical survey for evaluation of failure. Saudi Dent J, 26(2), 6367. doi:10.1016/j.sdentj.2014.02.003

Alothman, Y., \& Bamasoud, M. S. (2018). The Success of Dental Veneers According To Preparation Design and Material Type. Open Access Maced J Med Sci, 6(12), 2402-2408. doi:10.3889/oamjms.2018.353

Badel, T., Marotti, M., Pavicin, I. S., \& Basić-Kes, V. (2012). Temporomandibular disorders and occlusion. Acta Clin Croat, 51(3), 419-424.

Beier, U. S., Kapferer, I., Burtscher, D., \& Dumfahrt, H. (2012). Clinical performance of porcelain laminate veneers for up to 20 years. Int J Prosthodont, 25(1), 79-85.

Beier, U. S., Kapferer, I., \& Dumfahrt, H. (2012). Clinical long-term evaluation and failure characteristics of 1,335 all-ceramic restorations. Int J Prosthodont, 25(1), 70-78.

Calamia, J. R., \& Calamia, C. S. (2007). Porcelain laminate veneers: reasons for 25 years of success. Dent Clin North Am, 51(2), 399-417, ix. doi:10.1016/j.cden.2007.03.008

Carlsson, G. E. (2010). Some dogmas related to prosthodontics, temporomandibular disorders and occlusion. Acta Odontol Scand, 68(6), 313-322. doi:10.3109/00016357.2010.517412

Denissen, H. W., Wijnhoff, G. F., Veldhuis, A. A., \& Kalk, W. (1993). Five-year study of all-porcelain veneer fixed partial dentures. J Prosthet Dent, 69(5), 464-468. doi:10.1016/0022-3913(93)90154-g

Granell-Ruiz, M., Fons-Font, A., Labaig-Rueda, C., Martínez-González, A., Román-Rodríguez, J. L., \& Solá-Ruiz, M. F. (2010). A clinical longitudinal study 323 porcelain laminate veneers. Period of study from 3 to 11 years. Med Oral Patol Oral Cir Bucal, 15(3), e531-537. doi:10.4317/medoral.15.e531

Gresnigt, M. M. M., Cune, M. S., Schuitemaker, J., van der Made, S. A. M., Meisberger, E. W., Magne, P., \& Özcan, M. (2019). Performance of ceramic laminate veneers with immediate dentine sealing: An 11 year prospective clinical trial. Dent Mater, 35(7), 1042-1052. doi:10.1016/j.dental.2019.04.008

Gurel, G., Sesma, N., Calamita, M. A., Coachman, C., \& Morimoto, S. (2013). Influence of enamel preservation on failure rates of porcelain laminate veneers. Int J Periodontics Restorative Dent, 33(1), 31-39. doi:10.11607/prd.1488

Hui, K. K., Williams, B., Davis, E. H., \& Holt, R. D. (1991). A comparative assessment of the strengths of porcelain veneers for incisor teeth dependent on their design characteristics. Br Dent J, 171(2), 51-55. doi:10.1038/sj.bdj.4807602

Kundapur, P. P., Bhat, K. M., \& Bhat, G. S. (2009). Association of trauma from occlusion with localized gingival recession in mandibular anterior teeth. Dent Res J (Isfahan), 6(2), 71-74.

Layton, D. M., \& Clarke, M. (2013). A systematic review and meta-analysis of the survival of non-feldspathic porcelain veneers over 5 and 10 years. Int $J$ Prosthodont, 26(2), 111-124. doi:10.11607/ijp.3202

Layton, D. M., \& Walton, T. R. (2012). The up to 21 -year clinical outcome and survival of feldspathic porcelain veneers: accounting for clustering. Int $J$ Prosthodont, 25(6), 604-612.

Moher, D., Liberati, A., Tetzlaff, J., \& Altman, D. G. (2009). Preferred reporting items for systematic reviews and meta-analyses: the PRISMA statement. PLoS Med, 6(7), e1000097. doi:10.1371/journal.pmed.1000097

Nejatidanesh, F., Savabi, G., Amjadi, M., Abbasi, M., \& Savabi, O. (2018). Five year clinical outcomes and survival of chairside CAD/CAM ceramic laminate veneers - a retrospective study. J Prosthodont Res, 62(4), 462-467. doi:10.1016/j.jpor.2018.05.004

O'Carroll, E., Leung, A., Fine, P. D., Boniface, D., \& Louca, C. (2019). The teaching of occlusion in undergraduate dental schools in the UK and Ireland. Br Dent J, 227(6), 512-517. doi:10.1038/s41415-019-0732-6

Peumans, M., Van Meerbeek, B., Lambrechts, P., \& Vanherle, G. (2000). Porcelain veneers: a review of the literature. J Dent, $28(3)$, $163-177$. doi:10.1016/s0300-5712(99)00066-4

Racich, M. J. (2018). Occlusion, temporomandibular disorders, and orofacial pain: An evidence-based overview and update with recommendations. J Prosthet Dent, 120(5), 678-685. doi:10.1016/j.prosdent.2018.01.033 
Research, Society and Development, v. 10, n. 5, e7810514620, 2021

(CC BY 4.0) | ISSN 2525-3409 | DOI: http://dx.doi.org/10.33448/rsd-v10i5.14620

Rinke, S., Lange, K., \& Ziebolz, D. (2013). Retrospective study of extensive heat-pressed ceramic veneers after 36 months. J Esthet Restor Dent, 25(1), 42-52. doi:10.1111/jerd.12000

Sadowsky, S. J. (2006). An overview of treatment considerations for esthetic restorations: a review of the literature. J Prosthet Dent, 96(6), 433-442. doi:10.1016/j.prosdent.2006.09.018

Schmidt, K. K., Chiayabutr, Y., Phillips, K. M., \& Kois, J. C. (2011). Influence of preparation design and existing condition of tooth structure on load to failure of ceramic laminate veneers. J Prosthet Dent, 105(6), 374-382. doi:10.1016/s0022-3913(11)60077-2

Sheets, C. G., \& Taniguchi, T. (1990). Advantages and limitations in the use of porcelain veneer restorations. J Prosthet Dent, 64(4), 406-411. doi:10.1016/0022-3913(90)90035-b

Smales, R. J., \& Etemadi, S. (2004). Long-term survival of porcelain laminate veneers using two preparation designs: a retrospective study. Int J Prosthodont, 17(3), 323-326. 